\title{
Conditioned inhibitory effects of discriminated Pavlovian training with food in rats depend on interactions of search modes, related repertoires, and response measures
}

\author{
MATTHEW R. TINSLEY, WILLIAM TIMBERLAKE, and MATTHEW SITOMER \\ Indiana University, Bloomington, Indiana \\ and \\ DAVID R. WIDMAN \\ Juniata College, Huntingdon, Pennsylvania
}

\begin{abstract}
Like other accounts of conditioned inhibition, behavior systems predicts (and Experiment 1 shows) that during summation and retardation tests, presentation of a negative conditioned stimulus (a CS-) created by discriminative Pavlovian food conditioning will interfere with a focal search response, such as nosing in the feeder. Unlike most other views, behavior systems predicts (and Experiment 2 shows) that the same CS - can potentiate a general search response, like attending to a moving artificial prey stimulus. Contacting the prey stimulus in extinction increased over baseline when a CS - but not a CS Novel preceded it. Experiment 3 showed this effect was not due to unconditioned qualities of the CS- . It appears that the effects of a discriminative $\mathrm{CS}$ - depend on the interaction of the training contingency with search modes related to the unconditioned stimulus (US), their perceptual-motor repertoires and environmental support, and the choice of response measure.
\end{abstract}

The classic Rescorla-Wagner (1972) model accurately and efficiently predicts that a stimulus becomes inhibitory if it is followed by a smaller unconditioned stimulus (US) than predicted by the stimulus complex in which it is embedded. Although this view of conditioned inhibition (CI) accurately predicts numerous phenomena, it has several known difficulties. First, the Rescorla-Wagner model hypothesizes that $\mathrm{CI}$ and conditioned excitation are mediated by the same learning processes and develop in similar ways. Thus, repeated presentations of a negative conditioned stimulus ( $\mathrm{CS}-$ ) alone should result in extinction, just like repeated unreinforced presentations of a conditioned excitor. However, this does not appear to be true (Witcher \& Ayres, 1984;Zimmer-Hart \& Rescorla, 1974). Instead, extinction of CI is produced by extinction of the CS + with which the CS- was presented during inhibitory training (Best, Dunn, Batson, Meachum, \& Nash, 1985; Lysle \& Fowler 1985).

Second, because the Rescorla-Wagner (1972) model assumes excitation and inhibition are opposite poles of the same dimension, a stimulus should not produce simulta-

M.R.T. is now at the Department of Psychology, University of California, Los Angeles. This work is based upon research supported by the National Science Foundation under Grants 9408366 and 9817175 . We thank P. Clune and especially Joe Leffel for help, and Ralph Miller for advice. Correspondence should be addressed to W. Timberlake, Department of Psychology, Indiana University at Bloomington, 1101 East 10th Street, Bloomington, IN 47405 (e-mail: timberla@indiana.edu). neous excitatory and inhibitory effects. However, it has been shown in multiple procedures that the same stimulus can show excitatory and inhibitory effects under different test conditions (Droungas \& LoLordo, 1994; Matzel, Gladstein, \& Miller, 1988; Tait \& Saladin, 1986). Finally, because inhibition is assumed to act in opposition to excitation, presentation of a CS - should only reduce the probability of a response to the US or CS. However, several experiments have shown that presentation of a CS - can facilitate new behavior, such as withdrawal from a localized CS - (e.g., Hearst \& Franklin, 1977; Papini \& White, 1994; Wasserman, Franklin, \& Hearst, 1974). Because such new behavior is organized with respect to the CS - , it cannot be explained simply as the indirect result of the CS - inhibiting some other behavior (Dickinson, 1980).

The problem of a stimulus having both excitatory and inhibitory effects was addressed specifically by Miller and Schactman's (1985; see also Miller \& Matzel, 1988; Savastano, Cole, Barnet, \& Miller, 1999) comparator theory. Miller and Schactman proposed a theory of CI in which stimuli vary in terms of excitatory associative strength, and the animal's response to a CS - is determined by a comparison of the associative strength of the $\mathrm{CS}$ - with that of the comparator stimulus, the most salient and contiguous stimulus present during training. Rather than being the opposite of excitation, CI increases with the strength of the comparator's association with the US and decreases with the strength of the CS - association with the US. 
The comparator model has two important implications. First, typical inhibitory stimuli merely have less excitatory associative strength than the comparator. Second, inhibitory relationships do not accrue to a $\mathrm{CS}-$ as a result solely of its past associative training with the US, but as an outcome of current comparisons between the $\mathrm{CS}$ and other cues present during training, like the training context. The problem remains, though, of how to explain why the presentation of a conditioned inhibitor can produce emergent behavior in the conditioning sessions (Hearst \& Franklin, 1977; Papini \& White, 1994).

Affect-based theories handle the problem of emergent behavior by arguing that the CS - excites an opposing affective state to the $\mathrm{CS}+$. This affective state evokes its own hedonically opposite behavioral repertoire, which interferes with the criterion response (by producing withdrawal in the case of an appetitive CS +) (Amsel, 1992; Daly \& Daly, 1982; Dickinson, 1980; Wasserman et al., 1974). Although positing that opposing affective states conditioned to the CS - can explain some new responses (e.g., Wasserman et al., 1974), it does not explain the puzzle of why the same inhibitory stimulus can simultaneously produce both conditioned excitatory and inhibitory properties (Droungas \& LoLordo, 1994; Matzel et al., 1988; Tait \& Saladin, 1986).

In short, although there are several well-supported theories of CI, there is still room for an account that predicts excitatory and inhibitory effects of the same conditioned inhibitor and that explains the emergence of different behaviors in its presence. The candidate considered here is an extension of the behavior systems approach to CI procedures (see also Crawford \& Domjan, 1995). Like the affect-based account, the behavior systems approach hypothesizes that CI phenomena are related to the conditioning of different motivational states (modes) to the $\mathrm{CS}+$ and the $\mathrm{CS}-$, typically focal search to the CS+ and general search to the CS - . However, unlike the affect-based account, the behavior systems account hypothesizes that these modes fall along the same motivational dimension rather than an opposite affective dimension. Search modes simply are related to different search repertories and so support different responses related to the same US system (Timberlake, 1994, 2001; Timberlake \& Lucas, 1989). For example, the apparent avoidance of the CS - shown by pigeons could be the result of engaging in a looping-away locomotor pattern as part of a search for cues more proximate to food.

Research on Pavlovian response topography has supported the behavior systems hypothesis that CSs at different temporal distances from the US evoke different motivational modes (Akins, Domjan, \& Gutierrez, 1994; Fanselow \& Lester, 1988; Silva \& Timberlake, 1998; Timberlake \& Lucas, 1989). For instance, the behavior systems account of foraging for food (see reviews by Timberlake, 1983, 1990, 1993, 2001; Timberlake \& Lucas, 1989; Timberlake \& Silva, 1995) hypothesizes that the presentation of food entrains and conditions a sequence of excitatory search modes, making available their unique and species-typical perceptual-motor repertoires for further conditioning. At the level of search modes, the animal moves from a general search mode to a focal search mode as food becomes more imminent, expresses a handling/consuming mode when in contact with the food, and subsequently returns to a focal search mode, either finding food shortly or moving back to general search as food becomes less probable.

Analysis of appetitive CI procedures within the framework of a feeding behavior system (Timberlake, 2001) suggests that a short CS + that terminates with food reinforcement should become primarily associated with a focal search mode and with the responses related to the focal search repertoire that are best supported by the environment - for example, nosing in the feeder. In contrast, a CS - delivered at the interreward time maximally distant from food should become primarily associated with a general search mode and those responses from the general search repertoire best supported by the environment, such as locomotor search or attention to a potential prey stimulus.

An argument made against this account is that a CSpresented in the middle of a fixed-time (FT) interval should force the animal entirely out of the feeding system instead of conditioning a general search mode still related to food finding. However, several sources of data indicate that rats do not leave a motivational feeding system even with long intervals between food deliveries. For example, in a 24-h environment, rats on FT schedules imposing at least an 8-min interval between the availability of a 97-mg food pellet continue to show increased locomotor and exploratory behavior between pellets, as well as maintaining a short latency to pick up a food pellet when it drops (Lucas, Timberlake, \& Gawley, 1988). These data, along with others (e.g., Collier, 1983), indicate that rats determining their own total intake and meal size still search for food between pellets and eat similarsized continuous meals even when food delivery is very slow (Lucas \& Timberlake, 1988). Certainly, no less a tendency to persevere in general search behavior between food pellets would be expected of deprived rats limited to a single meal per day.

Thus, on the basis of the assumption that a differentially conditioned CS + and CS - are associated with different search modes and repertoires of the rat's feeding system, their presence should potentiate different and potentially competing responses. For example, general search behaviors potentiated by the CS - like locomotor search may interfere initially with highly focused focal search behaviors controlled by the CS + (such as sustained nosepoking in the feeder tray). Furthermore, it is worth noting that general search behaviors potentiated by the CS - will likely be different from the focal search responses typically measured.

In short, given a behavior system framework of conditioned search modes and related responses, it is possible that the performance decrement observed during ap- 
petitive CI testing may be due more to the differential potentiation of a general search mode and its related response repertoire than to direct inhibition of response associations, direct comparison of different amounts of CS-US excitation, or competition between opposite affective states. A behavior system view also points out that whether a putative conditioned inhibitor interferes with a response will depend on which response the experimenter measures. A particular CS will potentiate some responses and interfere with others, depending on the search mode it is associated with and the environmental support for the related repertoire.

Three studies reported in this paper examined different aspects of a behavior systems analysis. The first tested whether presentation of a $\mathrm{CS}-$, trained using singly alternating presentations of a $\mathrm{CS}+$ and a $\mathrm{CS}-$ in an FT schedule, reduced the focal search behavior of nosing in the feeder during summation and retardation tests. The second study tested directly whether presentation of a CS - trained as in Experiment 1 potentiated a general search mode and related responses, as measured by increased responding to the unconditioned general search probe of a rolling ball bearing. (Moving ball bearings appear to mimic aspects of unconditioned prey stimuli in rats; Timberlake, Wahl, \& King, 1982). The third experiment tested the extent to which the results of Experiment 2 could have been due to unconditioned rather than conditioned effects of the specific $\mathrm{CS}-$ and CS Novel used in that experiment.

\section{EXPERIMENT 1}

We expected training with a conditioned forward excitor to produce a CS + associated predominantly with a focal search mode and focal search responses, such as head-in-feeder. We expected the subsequent Pavlovian differential conditioning procedure to add a second focal search $\mathrm{CS}+$ and an unpaired stimulus $(\mathrm{CS}-$ ) associated predominantly with a general search mode and response repertoire. Following differential conditioning, presentation of the CS - during summation and retardation tests should potentiate a general search mode and response repertoire, thereby reducing the probability of responses related to a focal search mode. The result should be the typical CI-related reduction in measures of focal responding during summation and retardation tests. It is worth noting that although summation and retardation tests have been questioned as definitive of inhibition (Hallam, Matzel, Sloat, \& Miller, 1990; Papini \& Bitterman, 1993; Tait \& Saladin, 1986; Williams, Overmier, \& LoLordo, 1992), passing both tests nonetheless remains the most broadly accepted evidence that a stimulus functions as a conditioned inhibitor.

In the summation test, animals were presented in extinction with a compound of either CS + and CS - or CS + and CS Novel. CI was presumed present if responding to the $\mathrm{CS}+/ \mathrm{CS}-$ compound was less than responding to the CS+/CS Novel compound. In the retardation test the CS - was compared with a CS Novel in terms of the speed with which the two CSs became excitatory under conditions of new acquisition. CI was presumed present if responding to the $\mathrm{CS}-$ was less than responding to the CS Novel in the initial trials.

We used Pavlovian differential conditioning, rather than Pavlovian inhibitory conditioning or backward conditioning, because this procedure allowed a straightforward, temporally based behavior systems interpretation of the associations expected to form between the CSs and search modes. In appetitive differential conditioning, the CS + is closely paired with reinforcement whereas the CS - occurs in the middle of the interreinforcement interval. Thus, we expected this procedure to result in the CS+ primarily associated with a focal search mode and the CS - primarily associated with a general search mode.

\section{Method}

Subjects. We used 16 experimentally naive female SpragueDawley Norway rats (Rattus norvegicus), aged between 90 and 120 days. All the subjects were bred in the Department of Psychology animal colony and kept under a 12:12-h light:dark cycle with the lights off at $1800 \mathrm{~h}$. Subjects received ad-libitum water during the study and were maintained at $85 \%$ of ad-libitum body weight by restricting food intake.

Apparatus. We trained the subjects in four rectangular custombuilt aluminum chambers $(50 \mathrm{~cm}$ long $\times 35 \mathrm{~cm}$ wide $\times 30 \mathrm{~cm}$ high), each with a Plexiglas top and a slanted, clear Plexiglas front (along the long axis of the box), hinged at the bottom (see Silva $\&$ Timberlake, 1998, for a schematic). The recessed feeder $(3 \times 6 \times$ $2.5 \mathrm{~cm}$ deep, bottom edge $6 \mathrm{~cm}$ above the floor) was on one side wall of the chamber, located approximately $16 \mathrm{~cm}$ from the front wall. An infrared beam $1.5 \mathrm{~cm}$ above the front lip of the feeder and approximately $1 \mathrm{~cm}$ inside it was used to measure head-in-feeder responses. A jewel light and a small speaker (side by side) were located on either side of the feeder. Each was approximately $1.5 \mathrm{~cm}$ in diameter and located $7-8 \mathrm{~cm}$ above the floor. A small, weightsensitive floor platform, $10 \times 11 \mathrm{~cm}$, was directly in front of the feeder, and a second floor platform, $10 \times 24 \mathrm{~cm}$, was located near the middle of the back wall. Neither platform measure was used in the present experiment.

A sunken channel was located $12.5 \mathrm{~cm}$ from and parallel to the front long wall of each chamber. The channel was formed by two stainless steel rods placed approximately $1.6 \mathrm{~cm}$ below the floor of the chamber. Stainless steel ball bearings, $1.6 \mathrm{~cm}$ in diameter, could be released down this channel by operation of a BRS-Foringer pellet dispenser, modified to accept the ball bearings. Each ball bearing rolled into the chamber through a hole in the side wall opposite the feeder. If undisturbed, a bearing exited through the side wall containing the feeder approximately $4 \mathrm{sec}$ later (see Silva \& Timberlake, 1997, 1998). The floor was slanted toward the ball bearing channel from the front and back of the chamber, and both the channel and the floor were slanted approximately $7^{\circ}$ toward the feeder wall. As a result, even when the rats contacted and carried the bearing away from the channel, once the rat released it, the bearing rolled back to the channel and out of the chamber. Ball bearings were used in Experiments 2 and 3, but not in Experiment 1.

Pretraining. Prior to the study we restricted the subjects to a single daily feeding, adjusting the meal size until they attained $85 \%$ of their free-feeding weight. We maintained this feeding regime for the duration of the study. During the initial food restriction the subjects 
were weighed daily, allowing them to be gentled and to become used to handling and transportation in a rat carrier. Prior to training, subjects were provided with approximately $2 \mathrm{~g}$ of Noyes precision food pellets $(45 \mathrm{mg})$ so they could become accustomed to the pellets used during subsequent training. On each of the 2 days immediately prior to training, each subject received a 20-min exposure to the apparatus with no stimulus events programmed.

Feeder training. We gave all animals two sessions of feeder training on 2 successive days, with each session lasting $3 \mathrm{~min}$ or the presentation of 12 pellets, whichever occurred sooner. The experimenter placed a single food pellet in the feeder at the beginning of the session, and it was replaced automatically after being eaten, contingent on the animal removing its head from the feeder and moving off the platform in front of the feeder. The amount of time the animal had to remain away before the next pellet appeared was gradually increased to avoid rewarding the animal for remaining in or close to the feeder. We cleaned the apparatus between subjects using a dilute $(1: 200)$ Roccal-D detergent/deodorant solution.

Initial CS + training. To condition a transfer excitor for later summation testing, we trained all animals in a CS + conditioning procedure for 16 daily sessions, 6 days a week, with 12 training trials per session. Each trial consisted of the presentation of a 4-sec $2000-\mathrm{Hz}$ tone followed by two $45-\mathrm{mg}$ food pellets and a fixed, 96-sec interfood interval. During training, the frequency and duration of head-in-feeder during the CSs and during the intertrial interval (ITI) were recorded to establish asymptotic performance.

Differential conditioning. Following the initial CS + training, we trained the animals in a differential conditioning procedure for 16 sessions, with 12 training trials per session, across a 3-week period. We arranged the subjects in 4 subgroups of four (to allow counterbalancing of conditioned stimuli) in such a way as to minimize intergroup differences in the mean and variance of group body weight. Each trial consisted of the presentation of two stimuli, a $\mathrm{CS}+$ consisting of either a $4-\mathrm{sec}$ train of $3200-\mathrm{Hz}$ beeps $(0.3-\mathrm{sec}$ duration, 0.2 -sec intervals) or a 4-sec burst of white noise followed by two 45-mg food pellets, then a 44-sec interval, then presentation of the other stimulus as the CS- (a 4-sec burst of white noise or a $4-\mathrm{sec}$ train of $3200-\mathrm{Hz}$ beeps), then another $44-\mathrm{sec}$ interval. Hence, the CS + and CS - were singly alternating with a fixed 44-sec interval between them. This allowed us to fix the ITI at $96 \mathrm{sec}$. During training, the frequency and duration of head-in-feede $r$ responses during each CS were recorded.

Testing. Retardation and summation tests with the transfer excitor were carried out as part of an attempt to determine whether the training procedure produced reduced focal search responding during the CS- . The dependent variable was the time the animal spent interrupting an infrared beam by placing its head in the feeder during the CS presentation. Eight of the 16 trained animals were allocated to the retardation test; the remaining 8 animals were tested using a summation procedure (see below). In the retardation test each animal received 12 rewarded test trials per day over 2 days. The12 trials consisted of singly alternating presentations of a novel $\mathrm{CS}$ (4 sec of a $3-\mathrm{Hz}$ clicker) and the $\mathrm{CS}-$ from the discrimination training. Each stimulus was followed immediately by food. As in initial training, there was a fixed 96-sec ITI. The summation test animals received 12 extinction test trials per day alternating a compound CS composed of the transfer excitor CS + from the initial training (a 4-sec 2000-Hz tone) and the CS - from discrimination training or a compound CS composed of the transfer excitor CS+ from the initial training and the CS Novel (4 sec of a 3-Hz clicker). As in the retardation test, the compound stimuli were presented only once each $96 \mathrm{sec}$.

\section{Results and Discussion}

The left side of Figure 1 shows significant acquisition of total duration of head-in-feeder responding during initial training of the transfer excitor, averaged across four blocks of four daily trials $[F(3,45)=35.8, p<.01]$. The right side of Figure 1 shows rapid emergence of a discrimination be-

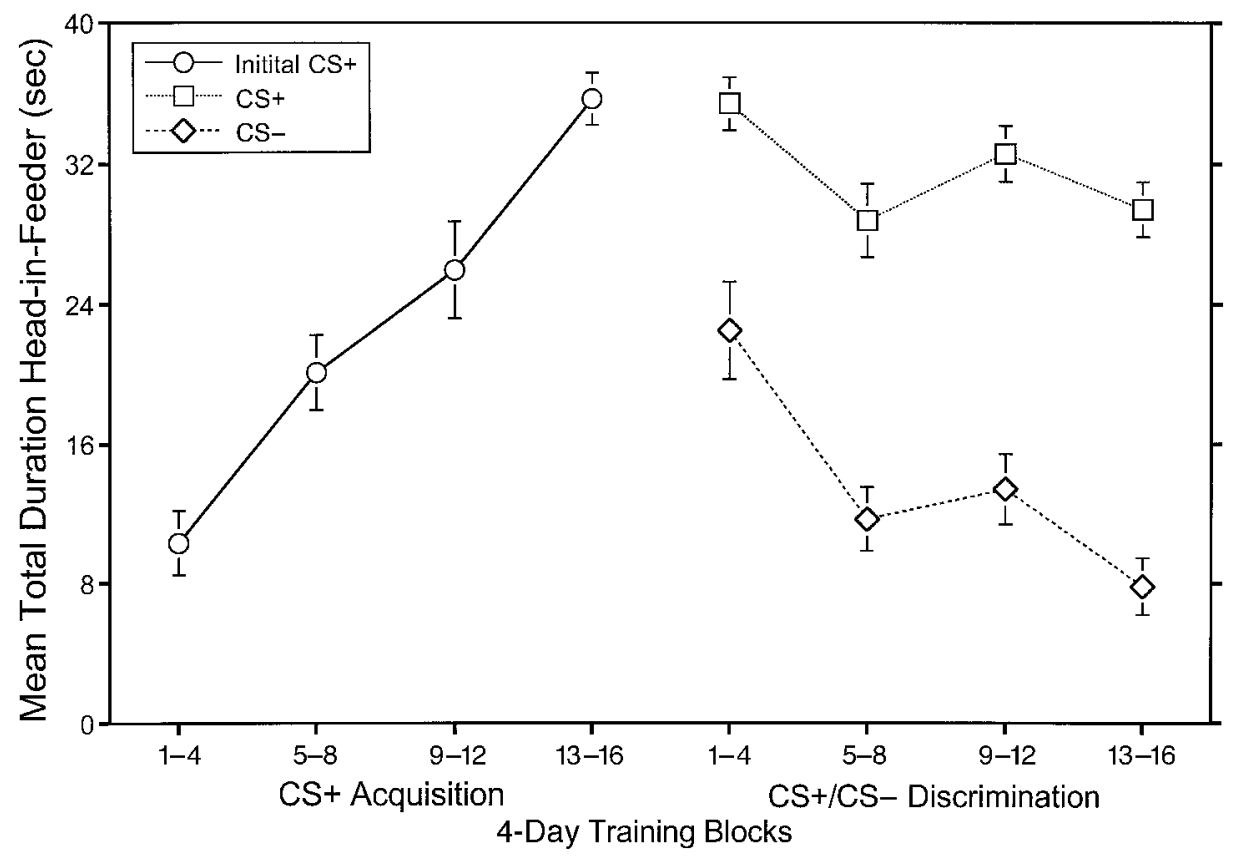

Figure 1. Mean total duration of head-in-feeder (sec) across 16 days of initial CS + (transfer excitor) training and 16 days of differential $\mathrm{CS}+/ \mathrm{CS}-$ training. Mean total durations were averaged by 4-day blocks of 12 daily presentations of the 4-sec CSs (see Experiment 1 results). 


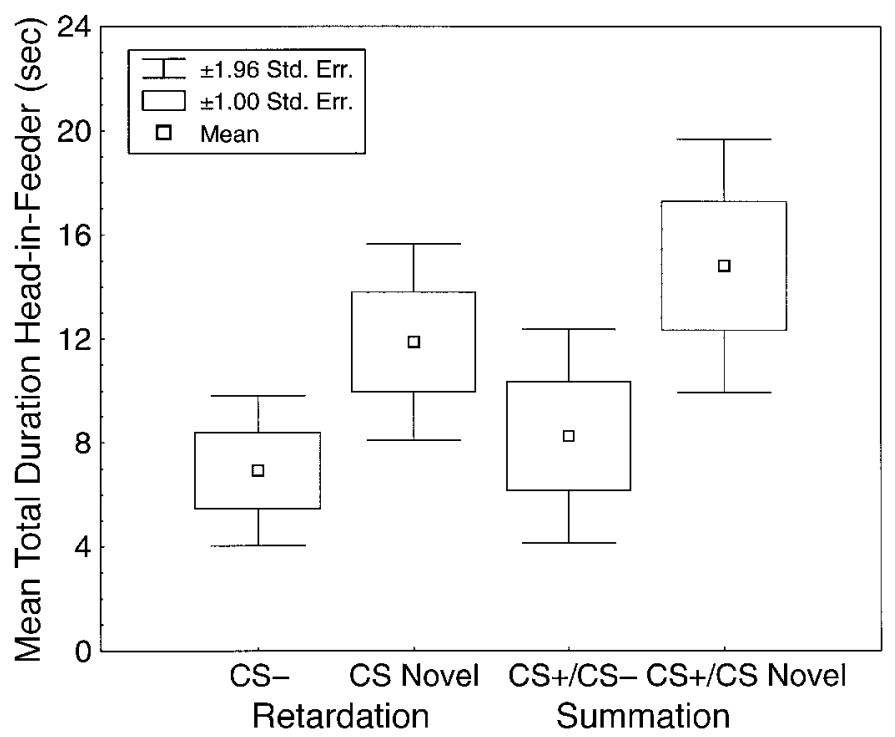

Figure 2. Mean total duration of head-in-feeder (sec) during summation and retardation trials on Test Day 1 for separate groups. Data were averaged over subjects for six presentations of each stimulus. In retardation tests, responding to the $\mathrm{CS}$ - was significantly lower than that to the CS Novel. In summation tests, responding to the $\mathrm{CS}+/ \mathrm{CS}-\mathrm{com}$ pound was significantly lower than that to the $C S+/ C S$ Novel compound (see Experiment 1 results).

tween $\mathrm{CS}+$ and $\mathrm{CS}-$ during the next four blocks of four daily sessions each $[F(1,15)=195.3, p<.01]$. There was also a significant trial-block effect and a trial-block $\times$ discriminative stimulus interaction $\left[F_{\mathrm{s}}(3,45)=19.0\right.$ and 8.10 , both $p$ s $<.01]$.

Figure 2 shows the results from the 1 st day of testing for the retardation and summation test groups. In the retardation group, we found significantly less total headin-feeder duration during the six CS - presentations than during the alternate six CS Novel presentations $[t(7)=$ $-2.40, p<.05]$. This difference was not present on the 2nd day $[t(7)=-1.04$, n.s.], and there was a significant overall increase in head-in-feeder from Day 1 to Day 2 $[F(1,7)=14.2, p<.01$ (data not shown) $]$. Similarly, the summation test group showed significantly less head-infeeder duration during presentation of the six CS+/CScompounds than during presentation of the alternate six $\mathrm{CS}+/ \mathrm{CS}$ Novel compounds on the 1 st day of testing $[t(7)=-3.09, p<.05]$, but not for the 2 nd day $[t(7)=$ -0.73 , n.s.].

These results show that $\mathrm{CS}-$ presentations reduced the total duration of head-in-feeder responding related to the focal search mode when compared with a novel stimulus during either new acquisition (the retardation test) or performance of previously conditioned responding in extinction (the summation test). Hence, we can conclude that the present conditioning procedure resulted in the outcomes typically characterizing CI: retardation of excitatory associations to the $\mathrm{CS}-$ when it serves as a new
$\mathrm{CS}+$ and reduction of excitatory responding to the original $\mathrm{CS}+$ in the presence of the $\mathrm{CS}-$. By showing both retardation and summation effects, these results strongly support the conclusion that our CS - training produced a classic conditioned inhibitor.

\section{EXPERIMENT 2}

The results of Experiment 1 established that our differential conditioning produced a conditioned inhibitor that reduces responding in summation tests and slows acquisition in retardation tests. These results were consistent with the traditional accounts of CI reviewed in the introduction as well as with the behavior systems view that the CS - conditions a general search state and responses that initially interfere with focal search behavior. The purpose of Experiment 2 was to test the uncommon behavior systems prediction that because a CSshould control a general search mode, it should potentiate related general search behavior.

To test for the presence of a general search mode and related behavior we used an unconditioned probe consisting of presentation of a ball bearing rolling down a channel that ran the length of the floor of the conditioning chamber, exiting near the feeder (see Silva \& Timberlake, 1997, 1998). The ball bearing probe procedure can be seen as analogous to testing for fear-potentiated startle by presenting an unconditioned startle stimulus (a loud sound) following presentation of a conditioned fear 
stimulus (Davis \& Astrachan, 1978). Similar to the way in which a loud sound is an unconditioned elicitor of startle, a moving ball bearing is an unconditioned elicitor of a sequence of predatory behaviors, including orientation, tracking, approach, and contact. If the differential Pavlovian procedure conditions a general search mode to the $\mathrm{CS}-$, then presenting the $\mathrm{CS}-$ prior to the ball bearing should increase predatory behavior toward the ball bearing relative to its preceding baseline level.

To clarify the effects of the CS - as well as to examine any arousal effects of a novel stimulus in evoking responding to the ball bearing, we also tested the effects on ball bearing contact relative to baseline of preceding the ball bearing with a novel CS. Instead of coding orientation to the bearing, we coded percent trials with a ball bearing contact (touching the ball bearing with the nose or paws). We used ball bearing contact because it has been documented to be part of a highly reliable predatory chain that begins with orient, and includes approach, chase, and capture (Timberlake et al, 1982). Most importantly, ball bearing contact is the simplest element for an observer to score reliably (reliability ratings are always .90 and above). We inferred the strength of the general search mode controlled by the CS - and the CS Novel by subtracting the unconditioned baseline of trials with a ball bearing contact from the trials with a contact following the CS- and the CS Novel.

\section{Method}

The number and provenance of naive subjects, apparatus, pretraining, feeder training, $\mathrm{CS}+$ acquisition, and $\mathrm{CS}+/ \mathrm{CS}-$ discrimination training were the same as for Experiment 1, except that discrimination training lasted 8 more days to ensure a separation between the $\mathrm{B}+$ and $\mathrm{C}-$ stimuli similar to those used in Experiment 1 .

Ball bearing baseline. Following differential Pavlovian conditioning, each animal received 6 days of ball bearing baseline. The animal was placed in the training chamber and presented with a rolling ball bearing every $96 \mathrm{sec}$ for a total of 12 trials. If the animal contacted the ball bearing with its nose or a paw during a trial, that trial was scored as a contact trial. Duration of contact was not scored, only relative frequency in the form of percent ball bearing trials with a contact. Percent trials with a contact (rather than total trials with a contact) was used because it allowed us to exclude the rare trials on which it was not possible to determine with certainty if the rat contacted the ball bearing. Although percent trials with a contact did not differ significantly over baseline trials, we have found the most stable individual baselines to be the median percent trials with a contact over the last three baseline trials (Days 4-6). The interobserver reliability in the present experiment, computed on a random sampling of baseline and test sessions, was $95.9 \%$. One animal was eliminated prior to testing because its baseline median of trials with a ball bearing contact was zero, thereby failing to reach our criterion of one contact.

Testing. The animals were tested in a single group of 15 in two sessions of 12 trials on successive days. A trial consisted of either a novel stimulus (a $3-\mathrm{Hz}$ clicker) or the $\mathrm{CS}-$ from training (either a 4 -sec burst of white noise or a train of $3200-\mathrm{Hz}$ beeps). Twelve trials singly alternated $4 \mathrm{sec}$ of the CS - and $4 \mathrm{sec}$ of the CS Novel. Two seconds into each 4-sec CS - or CS Novel stimulus, we released a ball bearing. Percent trials with a contact computed separately for $\mathrm{CS}-$ and $\mathrm{CS}$ Novel trials were compared with the baseline contact percentage to determine the effects of the CS- and CS Novel on ball bearing contact.

\section{Results and Discussion}

Figure 3 shows head-in-feeder over the final 4-day block of transfer excitor training, followed by six blocks

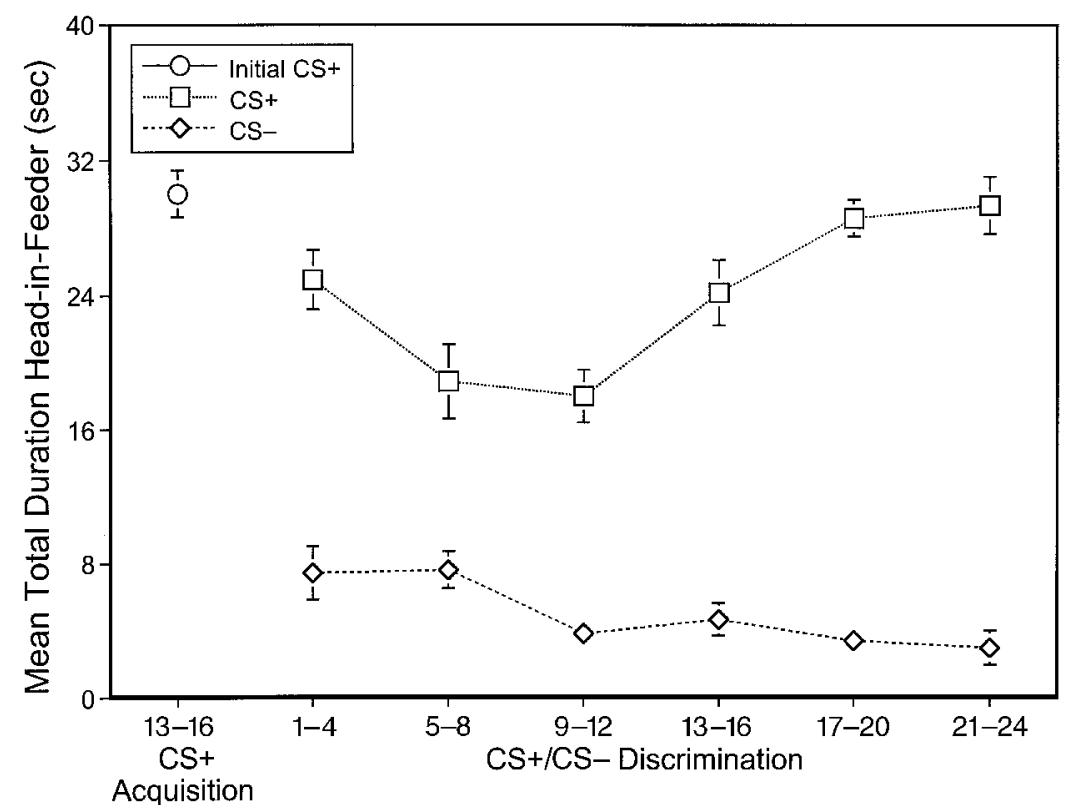

4-Day Training Blocks

Figure 3. Mean total duration of head-in-feeder during the last 4 days of $\mathrm{CS}+$ (transfer excitor) training and during the 24 days of differential CS $+/ C S-$ training. Mean total durations were averaged over 4-day blocks of 12 daily presentations of 4-sec CSs (see Experiment 2 results). 


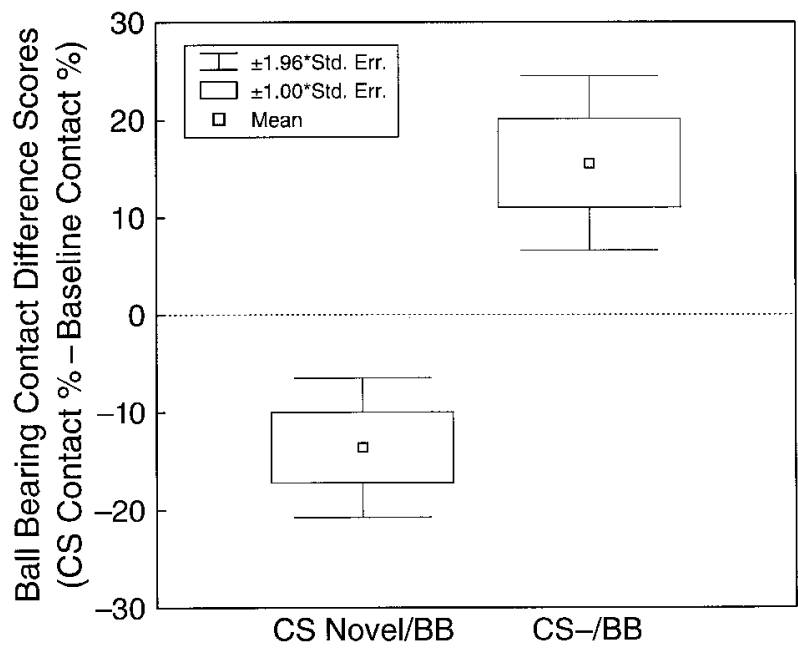

Figure 4. Mean change in percent trials with a ball bearing contact from baseline to the average of Test Days 1 and 2 as a function of presenting the ball bearing $2 \mathrm{sec}$ into the alternating CS - and CS Novel. The baseline for each subject was the median percent trials with a contact over the last 3 days of baseline (average median contact was $35.6 \% \pm 4.8 \%$ of trials). Data in Figure 4 were averaged over six daily presentations of each stimulus. The increase in bearing contacts shown following a $\mathrm{CS}-$ and the decrease in contacts following a CS Novel were both significant. CS Novel/BB, ball bearing contact following the CS Novel; CS - /BB, ball bearing contact following the CS - (see Experiment 2 results).

of discrimination training (CS $+/ \mathrm{CS}-$ ). Inspection of Figures 1 and 3 shows similar levels of asymptotic conditioning following both initial CS + (transfer excitor) training and subsequent $\mathrm{CS}+/ \mathrm{CS}-$ training. The trained transfer excitor was not tested in the present experiment, but we repeated the training procedure used in Experiment 1 to ensure similarity in training between the two experiments.

Figure 4 shows the difference in percent contact trials between the baseline and the average of the two test trials following the CS - and CS Novel. The mean baseline percent trials with a contact was $35.6( \pm 4.8 \mathrm{SE})$, computed as the average of individual medians of Baseline Days 4-6. A within-subjects $t$ test showed that presenting the CS - prior to presentation of a ball bearing significantly increased the average percent contact trials above the baseline level $[t(14)=3.10, p<.05]$, as we predicted. In contrast, presenting the CS Novel prior to presentation of a ball bearing significantly decreased the average percent trials with a contact below the baseline level $[t(14)=-3.86, p<.05]$. We assume that the latter effect was due to the initial disruptive effects of a novel stimulus on unconditioned approach to the ball bearings. As would be expected from these baseline comparisons, the CS - produced significantly greater percent trials with ball bearing contact than did the CS Novel $[t(14)=$ $4.63, p<.05]$.

In short, the data showed potentiation of ball bearing contact in the presence of the CS - relative to baseline, but no potentiation (in fact a reduction from baseline) of ball bearing contact in the presence of the CS Novel. Because we did not test the effects of the CS + on ball bearing contact in the present experiment, it might seem possible to argue that potentiation of ball bearing contact was not specific to the presentation of the CS-, but would have occurred to any stimulus that had been trained in the presence of food (like the CS + ). However, in addition to being counterintuitive given the high levels of head-in-feeder responding reported in Experiment 1 , our previous work using a differential conditioning procedure also showed potentiation of ball bearing contact by presentation of the $\mathrm{CS}-$, but a reduction of contact by the CS+ (see Figure 5.12 in Timberlake, 2001). Further, Silva and Timberlake (1997) twice showed that presenting a short CS + (previously trained alone) prior to a ball bearing probe stimulus decreased average contact of the bearing. Together these results support the view that increased contact with a ball bearing probe stimulus is not caused by the presence of just any conditioned or unconditioned $\mathrm{CS}$, but is related specifically to the presence of our CS-.

\section{EXPERIMENT 3}

The primary purpose of Experiment 3 was to rule out the possibility that the particular stimuli used as the $\mathrm{CS}-$ in Experiments 1 and 2 had unconditioned properties that were directly responsible for the increased ball bearing contacts following the CS- in Experiment 2 . The present experiment also tested whether unconditioned properties of the CS Novel, other than its unfamiliarity, may have contributed to the reduction in ball bearing contact when it was presented. To examine the unconditioned effects of the CS - and CS Novel, we used the same procedures of pretraining, ball bearing baseline, and testing used in Experiment 2, but we omitted $\mathrm{CS}+$ transfer excitor training and $\mathrm{CS}+/ \mathrm{CS}-$ discrimination training.

\section{Method}

Twelve naive female rats, 90-120 days old, served as subjects. The apparatus, pretraining, and feeder training were the same as in Experiments 1 and 2. Immediately following feeder training (and without any conditioning trials with food or presentations of the $\mathrm{CS}-$ ), each rat received 6 days of ball bearing baseline during which each was presented with a rolling ball bearing every $96 \mathrm{sec}$ for a total of 12 trials per day, the same baseline procedure used in Experiment 2. One rat was eliminated from the experiment following baseline because its median baseline trials with a contact over Days 4-6 of baseline was higher than $80 \%$, exceeding our unconditioned contact criterion. Following baseline the subjects were exposed to 4 days of the same testing conditions used in Experiment 2. Trials alternated between $4 \mathrm{sec}$ of the CS- from Experiment 2 (4 sec of white noise for half the rats and a train of $3200-\mathrm{Hz}$ beeps for the others) and $4 \mathrm{sec}$ of the CS Novel (a 3-Hz clicker). Each of these stimuli was followed $2 \mathrm{sec}$ after it began with the presentation of a ball bearing. The only way in which the testing conditions of Experiment 3 different from those of Experiment 2 (other than in using 4 rather than 2 test days) was the absence of any prior differential conditioning involving the $\mathrm{CS}-$. 


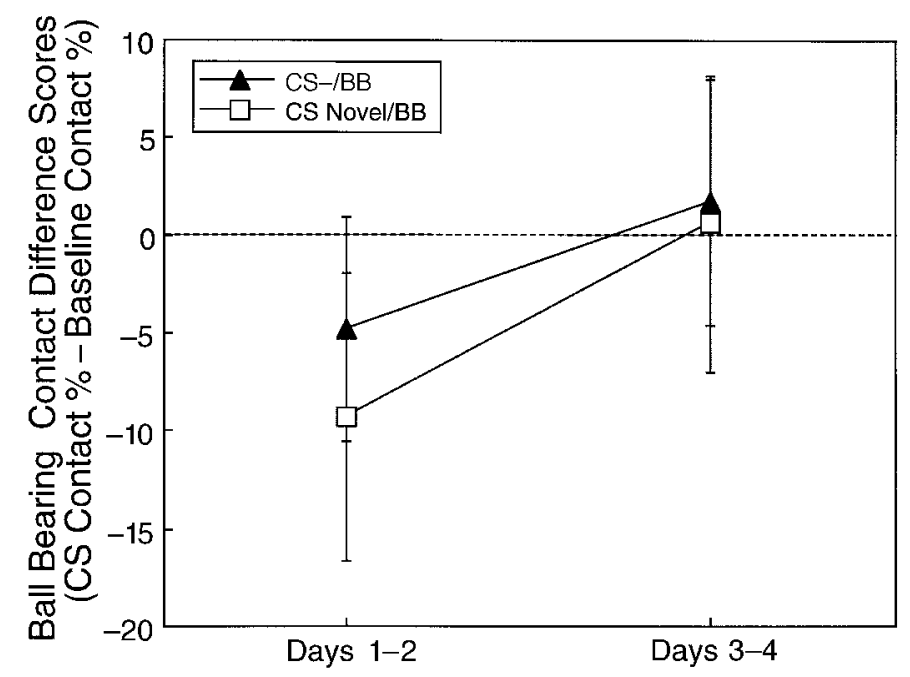

Figure 5. Mean change in percent trials with a ball bearing contact from baseline to test days as a function of presenting the ball bearing 2 sec into the CS - and CS Novel stimuli used in Experiment 2, but here presented in a pretest condition in the absence of food and without any excitatory or discriminative CS training. The baseline for each subject was the median percent trials with a contact over the last 3 days of baseline (mean baseline contact $=\mathbf{4 5 . 9 \%} \pm \mathbf{4 . 5 \%}$ of trials). Data were averaged over six daily presentations of each stimulus and across two 2-trial blocks. No differences singly or in combination were significant (see Experiment 3 results).

\section{Results and Discussion}

As can be seen in Figure 5, in the absence of any conditioning trials, presenting either the unconditioned CS - or the CS Novel from Experiment 2 failed to increase contact of the ball bearing over baseline levels (mean baseline contact was 45.9 ( $\pm 4.5 \mathrm{SE})$. Analysis of variance showed neither a CS-type effect nor a days effect $\left[F_{\mathrm{S}}(1,10)<1\right.$ and 3.95 , n.s. $]$. Inspection of the figure raised the possibility that the CS Novel decreased baseline ball bearing contact during the first 2 days of its presentation, but a within-subjects $t$ test was not significant $[t(10)=1.35$, n.s.]. Similar $t$ values for other days and groups were all less than one. It is worth noting that the average baseline contact level in the present experiment was not significantly different from the baseline level in Experiment $2[t(24)=1.50, p>.10]$.

When combined with the results of Experiment 2, the present results do not support the hypothesis that unique unconditioned aspects of the CS - were responsible for the increase in ball bearing contact. Although the data do not completely eliminate the possibility that unconditioned qualities of the $\mathrm{CS}-$ may have interacted uniquely with the training procedures, it is worth noting that no comparison of responding to the two CS - in any condition or experiment showed a significant difference, making unique interactions with particular conditioning less likely. These data appear most compatible with the argument that a novelty reaction to the CS Novel tended to decrease responding to ball bearings that were presented in its presence during the first two test trials.
In short, the increase in ball bearing contact above baseline produced by the CS - in Experiment 2 (and in Timberlake, 2001) was most likely due to previous Pavlovian discrimination training. It was not dependent on unconditioned qualities of the $\mathrm{CS}-$, or related to the initial decremental effect of the CS Novel. It would have been appropriate, though, to expose subjects to the CS Novel prior to testing to reduce novelty reactions.

\section{GENERAL DISCUSSION}

According to the present data, the effects of a conditioned inhibitor varied as a function of the testing procedures and the response that was measured. The same CS - had decremental effects on a previously conditioned response and on new conditioning as a $\mathrm{CS}+$, while it also had incremental effects on an unconditioned response. Rephrased, presentation of a conditioned inhibitor in summation and retardation tests resulted in a reduction in the focal search behavior of nosing in the feeder, whereas presentation of a conditioned inhibitor prior to an unconditioned ball bearing probe for general search increased orientation to and contact of that probe. Viewed in this way, our results are compatible with predictions of the behavior systems approach that presentation of a discriminated conditioned inhibitor will initially interfere with a focal search mode and related behaviors and potentiate a general search mode and related behaviors.

Regardless of theoretical interpretation, the present results are not well addressed by theories of CI suggest- 
ing that inhibition is the opposite of excitation (Rescorla $\&$ Wagner, 1972) and are not compatible with those suggesting that inhibitors primarily act as stimuli of opposite motivational significance to excitors (Amsel, 1992; Daly \& Daly, 1982; Dickinson, 1980; Wasserman et al., 1974). The former view does not explain how a conditioned inhibitor facilitates emergent behaviors, and the opposite motivation view appears to rule out the possibility that our CS - could facilitate attention and approach to an unconditioned artificial prey stimulus. Miller and Schactman's (1985) comparator theory is close to the present account in that it argues that conditioned inhibitors also establish excitatory associations to the US and that their inhibitory effects are relative to the context. However, the present form of the comparator hypothesis does not address the effects of a conditioned inhibitor on emergent behaviors, and, so, at present does not account for the potentiation of behavior directed at the ball bearing by presentation of the CS- .

It might be possible to make a simple attentional interpretation of these data (Mackintosh, 1983). If we assume the CS - switches attention away from the feeder, then it could be argued that the unconditioned rolling ball bearing became the most salient stimulus in the chamber, thereby attracting the increased contact behavior we report. However, an attentional hypothesis also would seem to predict that the highest frequency of contact with the ball bearing should occur during baseline, because baseline had the fewest competing stimuli. In the test phase, when other stimuli are present, there should be reduced attention to the unconditioned moving stimulus and thus reduced contact. In contrast to these predictions, our results showed increased contact during the CS - pairings relative to baseline.

Another argument is that because the inhibitory summation and retardation effects of the CS - in Experiment 1 were judged relative to the CS Novel, they may have been due less to the inhibitory effects of the CSthan to unique unconditioned excitatory qualities of the CS Novel. The possibility that the uniqueness of the CS Novel produced the present effect comes from the fact that it was not counterbalanced with the CS-; we chose instead to counterbalance the latter with the CS + in conditioning to rule out unique learning interactions affecting ball bearing behavior in Experiment 2. It is important to emphasize that any potential unique unconditioned effects of our CS Novel were not responsible for the excitatory effect of the CS - on ball bearing contact in Experiment 2 because the $\mathrm{CS}-$ effect was judged against an independent baseline of ball bearing contact, not against the level of ball bearing contact following the CS Novel. In addition, the results of both Experiments 2 and 3 suggest that the CS Novel had an unconditioned inhibitory rather than an excitatory quality. Such an inhibitory quality would have tended to mask rather than highlight the inhibitory effect of the CS - in the retardation and summation tests.
It also has been proposed that the $\mathrm{CS}-$ produced active withdrawal from the area of the feeder, thereby producing, as a side effect, an increase in ball bearing contact because withdrawal placed the animal nearer to the ball bearing. Three things are important to note. First, withdrawal from the feeder does not affect the distance of the animal from the ball bearing channel. The feeder is at one end of the chamber. The ball bearing enters through the opposite end of the chamber and rolls the length of the chamber toward the feeder, exiting less than $10 \mathrm{~cm}$ from it. Second, no location in the chamber is more than a body's length from the ball bearing channel. Third, the average amount of time with head-in-feeder in the ball bearing test was actually slightly (though not significantly) larger during the CS - than during the CS Novel, an outcome suggesting competition with rather than facilitation of attention to the ball bearing. In short, there is no suggestion that the CS- produced differential withdrawal from the feeder, and, on the basis of the structure of the apparatus, no way that withdrawal would have changed the proximity of the ball bearing to the rat.

Finally, it has been suggested that the results of Experiments 1 and 2 could have arisen because the CShad complex arousing properties that interfered with both responding to the transfer excitor in summation and the acquisition of new conditioning during retardation, while also increasing ball-bearing-related behavior following presentation of the $\mathrm{CS}-$ in the ball bearing probe test. Because Experiment 3 failed to show that presentation of the unconditioned CS - increased ball bearing contact, it appears that the arousing properties shown in Experiments 1 and 2 must depend on the transfer excitor and/or CS +/CS - discrimination training. If so, this post hoc arousal argument appears indistinguishable from the a priori and more specific behavior systems view we tested.

According to the behavior systems view, the results of Experiment 1 and 2 arose because of the conditioning of a general search mode and relevant responses to the $\mathrm{CS}-$ in training. Conditioned and unconditioned responses related to the general search repertoire evoked by the CS - interfered with focal search response to the $\mathrm{CS}+$ excitor (the summation effect) and to the acquisition of focal search responses by the CS - in new conditioning (the retardation effect). At the same time, the priming of the general search repertoire by the CS- facilitated reactions to the ball bearing in the unconditioned probe test.

In addition to predicting the present results, a behavior systems approach is consistent with several puzzling findings in the CI literature. Although the basic Rescorla and Wagner (1972) model assumed that stimuli acquire excitation and inhibition in similar ways, repeated presentations of the CS + in the training context and in the absence of any US results in notably better extinction of the decremental effects of the $\mathrm{CS}-$ than do repeated presentations of the CS - alone (Best et al., 1985; Lysle \& 
Fowler, 1985). A behavior systems approach accounts for this effect because the general search state presumed to underlie the effects of the CS- depends on the presence of food in the situation. It is worth pointing out, though, that the effect of withdrawing the US should have less effect on the general search excitatory effects of the CS - than on the focal search excitatory effects of the CS+ because the general search mode and related repertoire are less dependent on reliable presentation of food than the focal search mode and related repertoire (Timberlake, 2001). It would also follow that extinction of the CS+ outside the training context, or extinction based on unpaired CS + and US presentations, should minimally alter the effects of presenting a CS- .

The present account also appears compatible with data showing that conditioned inhibitors support multiple associations, either simultaneously or in different contexts (Droungas \& LoLordo, 1994; Matzel et al., 1988; Tait \& Saladin, 1986). The behavior systems account has the potential advantage of predicting a priori the kinds of appetitive behaviors that will be potentiated or interfered with by a conditioned inhibitor. Finally, a behaviorsystems-based approach is consistent with the recent finding that a second-order stimulus that precedes a backward conditioned inhibitory stimulus will increase rather than decrease the typical criterion behavior (Barnet \& Miller, 1996). Comparator theory predicts this outcome by adding to conditioning an explicit timing process that encodes the time of each stimulus with respect to the original position of the US. A behavior systems account depends more on the relative time information that regulates the balance among expression of the different search modes (Silva \& Timberlake, 1997). Thus, a second stimulus that predicts a previously conditioned backward CS will fall in the range of expression of the prefood focal search mode and thus should potentiate focal search behaviors, such as nosing in the feeder.

The mechanism that underlies conditioned inhibition has been debated since Pavlov's (1927) experiments. Most of the successful accounts have assumed a primary excitatory/inhibitory dimension of associations and/or motivational states and have labeled as conditioned inhibitors those stimuli that after training decrease apparently excitatory criterion responses. Konorski (1967) and Wagner and Brandon (1989) proposed multiple forms of associations related to conditioned inhibitors. Comparator theory dissolved the strict link between behavior and positive and negative associations by suggesting that stimuli producing a decrement could be merely less excitatory rather than necessarily inhibitory (Miller \& Matzel, 1988); however, it has little to say about the emergent behavior to a conditioned inhibitor.

The behavior systems approach (see also Fanselow \& Lester, 1988) adds a more complex motivational framework that interacts with conditioning contingencies to account for relative effects of conditioned inhibitors as well as predicting the emergence or enhancement of behaviors related to the search mode and system under investigation. However, it follows comparator theory in making use of the environment, especially in the case of conditioning general search states (Timberlake, 1986), as well as in the role of the environment in supporting specific elements of the search repertoires. How comprehensive the behavior systems approach can be and how well it covers the strengths of more traditional models has yet to be established.

\section{REFERENCES}

Akins, C. A., Domjan, M., \& Gutierrez, G. (1994). Topography of sexually conditioned behavior in male Japanese quail (Coturnix japonica) depends on the CS-US interval. Journal of Experimental Psychology: Animal Behavior Processes, 20, 199-209.

Amsel, A. (1992). Frustration theory: An analysis of dispositional learning and memory. Cambridge: Cambridge University Press.

Barnet, R. C., \& Miller, R. R. (1996). Second-order excitation mediated by a backward conditioned inhibitor. Journal of Experimental Psychology: Animal Behavior Processes, 22, 279-296.

Best, M. R., Dunn, D. P., Batson, J. D., Meachum, C. L. \& Nash, S. M. (1985). Extinguishing conditioned inhibition in flavor aversion learning: Effects of repeated testing and extinction of the excitatory element. Quarterly Journal of Experimental Psychology, 37B, 359378.

Collier, G. (1983). Life in a closed economy: The ecology of learning and motivation. In M. D. Zeiler \& P. Harzem (Eds.), Advances in analysis of behaviour: Vol. 3. Biological factors in learning (pp. 223274). London: Wiley.

Crawford, L. L., \& Domuan, M. (1995). Conditioned inhibition of social approach in male Japanese quail (Coturnix japonica) using visual exposure to a female. Behavioural Processes, 36, 163-169.

DALY, H. B., \& DALY, J. T. (1982). A mathematical model of reward and aversive nonreward: Its application in over 30 appetitive learning situations. Journal of Experimental Psychology: General, 111, 441-480.

Davis, M., \& Astrachan, D. I. (1978). Conditioned fear and startle magnitude: Effects of different footshock or backshock intensities used in training. Journal of Experimental Psychology: Animal Behavior Processes, 4, 95-103.

DiCKINSON, A. (1980). Contemporary animal learning theory. Cambridge: Cambridge University Press.

Droungas, A., \& LoLordo, V. M. (1994). Evidence for simultaneous excitatory and inhibitory associations in the explicitly unpaired procedure. Learning \& Motivation, 25, 1-25.

FANSELOW, M. S., \& LESTER, L. S. (1988). A functional behavioristic approach to aversively motivated behavior: Predatory imminence as a determinant of the topography of defensive behavior. In R. C. Bolles \& M. D. Beecher (Eds.), Evolution and learning (pp. 185212). Hillsdale, NJ: Erlbaum.

Hallam, S. C., Matzel, L. D., Sloat, J. S., \& Miller, R. R. (1990). Excitation and inhibition as a function of post training extinction of the excitatory cue used in Pavlovian inhibition training. Learning \& Motivation, 21, 59-84.

Hearst, E., \& Franklin, S. R. (1977). Positive and negative relations between a signal and food: Approach-withdrawal behavior to the signal. Journal of Experimental Psychology: Animal Behavior Processes, 3, 37-52.

KONORSKI, J. (1967). Integrative activity of the brain: An interdisciplinary approach. Chicago: University of Chicago Press.

Lucas, G. A., \& Timberlake, W. (1988). Interpellet delay and meal patterns in the rat. Physiology \& Behavior, 43, 259-264.

Lucas, G. A., Timberlake, W., \& Gawley, D. J. (1988). Adjunctive behavior in the rat under periodic food delivery in a 24-hour environment. Animal Learning \& Behavior, 16, 19-30.

Lysle, D. T., \& Fowler, H. (1985). Inhibition as a "slave" process: Deactivation of conditioned inhibition through extinction of conditioned excitation. Journal of Experimental Psychology: Animal Behavior Processes, 11, 71-94.

Mackintosh, N. J. (1983). Conditioning and associative learning. New York: Oxford University Press.

Matzel, L. D., Gladstein, L., \& Miller, R. R. (1988). Conditioned 
excitation and conditioned inhibition are not mutually exclusive. Learning \& Motivation, 19, 99-121.

Miller, R. R, \& MAtzel, L. D. (1988). The comparator hypothesis: A response rule for the expression of associations. In G. H. Bower (Ed.), The psychology of learning and motivation: Advances in research and theory (Vol. 22, pp. 51-92). San Diego: Academic Press.

Miller, R. R, \& Schactman, T. R. (1985). Conditioning context as an associative baseline: Implications for response generation and the nature of conditioned inhibition. In R. R. Miller \& N. E. Spear (Eds.), Information processing in animals: Conditionedinhibition (pp. 51-88). Hillsdale, NJ: Erlbaum.

Papini, M. R., \& Bitterman, M. E. (1993). The two-test strategy in the study of inhibitory conditioning. Journal of Experimental Psychology: Animal Behavior Processes, 19, 342-352.

PAPINI, M. R., \& White, N. (1994). Performance during signals for reward omission. Learning \& Motivation, 25, 45-64.

Pavlov, I. P. (1927). Conditioned reflexes (G. V. Anrep, Trans.). London: Oxford University Press.

Rescorla, R. A. \& Wagner, A. R. (1972). A theory of Pavlovian conditioning. Variations in the effectiveness of reinforcement and nonreinforcement. In A. H. Black \& W. F. Prokasy (Eds.), Classical conditioning II: Current research and theory (pp. 64-69). New York: Appleton-Century-Crofts.

Savastano, H. I., Cole, R. P., Barnet, R. C., \& Miller, R. R. (1999). Reconsidering conditioned inhibition. Learning \& Motivation, 30, 101-127.

Silva, K. M., \& Timberlake, W. (1997). A behavior systems view of conditioned states during long and short CS-US intervals. Learning \& Motivation, 28, 465-490.

Silva, K. M., \& Timberlake, W. (1998). A behavior systems view of responding to probe stimuli during an interfood clock. Animal Learning \& Behavior, 26, 313-325.

TAIT, R. W., \& Saladin, M. E. (1986). Concurrent development of excitatory and inhibitory associations during backward conditioning. Animal Learning \& Behavior, 14, 133-137.

Timberlake, W. (1983). Rats' responses to a moving object related to food or water: A behavior-systems analysis. Animal Learning \& Behavior, 11, 309-320.

TimberlaKe, W. (1986). Unpredicted food produces a mode of behavior that affects rats' subsequent reactions to a conditioned stimulus: A behavior-system approach to "context blocking." Animal Learning \& Behavior, 14, 276-286.

Timberlake, W. (1990). Natural learning in laboratory paradigms. In
D. A. Dewsbury (Ed.), Contemporary issues in comparative psychology (pp. 31-54). Sunderland, MA: Sinauer.

TimberlaKe, W. (1993). Behavior systems and reinforcement: An integrative approach. Journal of the Experimental Analysis of Behavior, 60, 105-128.

Timberlake, W. (1994). Behavior systems, associationism, and Pavlovian conditioning. Psychonomic Bulletin \& Review, 1, 405-420.

Timberlake, W. (2001). Motivational modes in behavior systems. In R. R. Mowrer \& S. B. Klein (Eds.), Handbook of contemporary learning theories (pp. 155-209). Hillsdale, NJ: Erlbaum.

Timberlake, W., \& LuCAS, G. A. (1989). Behavior systems and learning: From misbehavior to general principles. In S. B. Klein \& R. R. Mowrer (Eds.), Contemporary learning theories: Instrumental conditioning theory and the impact of biological constraints on learning (pp. 237-275). Hillsdale, NJ: Erlbaum.

Timberlake, W., \& Silva, K. M. (1995). Appetitive behavior in ethology, psychology, and behavioral systems. In N. S. Thompson (Ed.), Perspectives in ethology (Vol. 11, pp. 211-253). New York: Plenum.

Timberlake, W., Wahl, G., \& King, D. (1982). Stimulus and response contingencies in the misbehavior of rats. Journal of Experimental Psychology: Animal Behavior Processes, 8, 62-85.

Wagner, A. R., \& Brandon, S. E. (1989). Evolution of a structured connectionist model of Pavlovian conditioning (AESOP). In S. B. Klein \& R. R. Mowrer (Eds.), Contemporary learning theories: Pavlovian conditioning and the status of traditional learning theory (pp. 149-189). Hillsdale, NJ: Erlbaum.

Wasserman, E. A., Franklin, S. R., \& Hearst, E. (1974). Pavlovian appetitive contingencies and approach versus withdrawal to conditioned stimuli in pigeons. Journal of Comparative \& Physiological Psychology, 86, 616-627.

Williams, D. A., Overmier, J. B., \& LoLordo, V. M. (1992). A reevaluation of Rescorla's early dictums about Pavlovian conditioned inhibition. Psychological Bulletin, 111, 275-290.

Witcher, E. S., \& Ayres, J. J. B. (1984). A test of two methods for extinguishing Pavlovian conditioned inhibition.Animal Learning \& Behavior, 12, 149-156.

Zimmer-Hart, C. L., \& Rescorla, R. A. (1974). Extinction of Pavlovian conditioned inhibition. Journal of Comparative \& Physiological Psychology, 86, 837-845.

(Manuscript received September 6, 2001; revision accepted for publication April 9, 2002.) 